\title{
Improving the prediction of effective lens position for intraocular lens power calculations
}

\author{
Juanita N. Chui ${ }^{1,2}$, Keith Ong ${ }^{2,3}$ \\ 'Sydney Medical School, The University of Sydney, New South Wales, Australia; \\ ${ }^{2}$ Royal North Shore Hospital, Kolling Institute, St Leonards, New South Wales, \\ Australia; ${ }^{3}$ Department of Ophthalmology, Northern Clinical School, Faculty of \\ Medicine, University of Sydney, New South Wales, Australia
}

\begin{abstract}
Purpose: Achieving the desired post-operative refraction in cataract surgery requires accurate calculations for intraocular lens (IOL) power. Latest-generation formulae use anterior-chamber depth (ACD) - the distance from the corneal apex to the anterior surface of the lens - as one of the parameters to predict the post-operative IOL position within the eye, termed the effective lens position (ELP). Significant discrepancies between predicted and actual ELP result in refractive surprise. This study aims to improve the predictability of ELP. We hypothesise that predictions based on the distance from the corneal apex to the mid-sagittal plane of the cataractous lens would more accurately reflect the position of the principal plane of the non-angulated IOL within the capsular bag. Accordingly, we propose that predictions derived from $A C D+1 / 2 L T$ (length thickness) would be superior to those from ACD alone.

Design: Retrospective cohort study, comparing ELP predictions derived from ACD to a proposed prediction parameter.

Method: This retrospective study includes data from 162 consecutive cataract surgery cases, with posterior-chamber IOL (AlconSN60WF) implantation. Pre- and postoperative biometric measurements were made using the IOLMaster700 (ZEISS, Jena, Germany). The accuracy and reliability of ELP predictions derived from ACD and ACD + $1 / 2 L T$ were compared using software-aided analyses.

Results: An overall reduction in average ELP prediction error $\left(P E_{E L P}\right)$ was achieved using the proposed parameter (root-mean-square-error [RMSE] $=0.50 \mathrm{~mm}$ ), compared to $A C D$ $(R M S E=1.57 \mathrm{~mm})$. The mean percentage $P E_{E L P}$ comparing between eyes of different axial lengths, was $9.88 \% \pm 3.48 \%$ and $-34.9 \% \pm 4.79 \%$ for predictions derived from $A C D+1 / 2 L T$ and ACD, respectively. A 44.10\% $\pm 5.22 \%$ mean of differences was observed $(p<0.001)$. Conclusion: $A C D+1 / 2 L T$ predicts ELP with greater accuracy and reliability than $A C D$ alone; its use in IOL power calculation formulae may improve refractive outcomes.
\end{abstract}

Keywords: anterior-chamber depth, cataract, cataract surgery, ELP, effective lens position, intraocular lens, IOL power, IOL power calculation formulae

Correspondence: Dr. Keith Ong, 2 Railway Avenue, Eastwood, New South Wales 2122, Australia.

E-mail: keithong@optusnet.com.au 


\section{Introduction}

In cataract surgery, achieving the desired post-operative refraction depends on selecting the appropriate intraocular lens (IOL) power. In IOL power calculation formulae, corneal curvature, axial length $(\mathrm{AL})$ and the post-operative position of the IOL implant within the eye, referred to as the effective lens position (ELP), play an important part in the prediction of the refractive outcome. While ocular biometrics can be measured pre-operatively, the post-operative ELP is predicted. Many formulae have been developed to predict the optical properties of the pseudophakic eye, yet accurately and reliably predicting ELP remains a challenge in modern IOL power calculations. ${ }^{1}$ As such, improving the predictability of ELP should minimise refractive surprise and thereby improve refractive outcomes.

In IOL power calculations, it has been shown that inaccuracy in predictions of post-operative ELP represent one of the greatest sources of total refractive prediction error. ${ }^{1}$ Many modern IOL power calculation formulae, including Holladay II, Olsen, Barrett Universal II and Haigis, use anterior-chamber depth (ACD) as one of the parameters to predict post-operative ELP.

The ACD measurement in optical biometry is defined by the distance from the apex of the cornea to that of the lens. ${ }^{2}$ However, the principal plane of the non-angular biconvex IOL, in its intended position, should lie in the mid-sagittal plane of the capsular bag. In the phakic eye, this position approximates the mid-sagittal plane of the cataractous lens. This deviation from the theoretical post-operative position of the IOL may contribute to discrepancy between the predicted ELP based on ACD alone and the post-operative outcome, particularly when the IOL tends to be markedly thinner than the cataractous lens. Furthermore, the distance of the anterior surface of the lens from the corneal apex is subject to variations in lens thickness (LT) due to aging and pathology, ${ }^{3-5}$ which may compromise ELP predictions.

In current methods, a range of assumptions, correction factors and optimisation processes are used to account for prediction error due to ocular biometric measurements and limitations of $\mathrm{IOL}$ power calculation formulae. ${ }^{6}$ However, such assumptions bear an inherent degree of inaccuracy and optimisation of constants for regression minimises average error at best. Furthermore, predictions for post-operative refraction are known to vary between different IOL formulae. Not only does this contribute to the challenge in selecting the appropriate IOL power for cataract surgery, but to refractive surprise as well.7 Such variation in predictions have been reported to be most prevalent in non-average eyes, such that those with extreme myopia and extreme hypermetropia are particularly prone to refractive surprise. ${ }^{8,9}$

The present study aims to develop an algorithm to predict ELP with improved accuracy and precision for use in IOL power calculations. We propose an 
approach based on structural geometry and anatomy of the anterior chamber of the eye rather than statistical associations between pre- and post-operative ocular biometric factors, as seen in many preceding IOL power calculation formulae.

\section{Hypothesis and proposed ELP prediction parameter}

ELP defined as the distance from the anterior apex of the cornea to the mid-sagittal plane of the IOL optic may provide a more accurate model for prediction (Fig. 1). The position of the mid-sagittal plane of the phakic lens should closely approximate the principal plane of the non-angulated biconvex IOL within the capsular bag. Accordingly, we hypothesise that accounting for half the thickness of the cataractous lens in addition to ACD, according to the following algorithm, will help improve predictions for ELP: ACD + 1/2LT.

Lens growth continues throughout life, such that ACD decreases as LT increases with age. ${ }^{5}$ However, the distance from the corneal apex to the mid-sagittal plane of the cataractous lens should be relatively unchanged. This model should remove variation due to $L T$ and more closely reflects the position of the $I O L$, in comparison to $A C D$, the predominant parameter for ELP prediction in current methods.

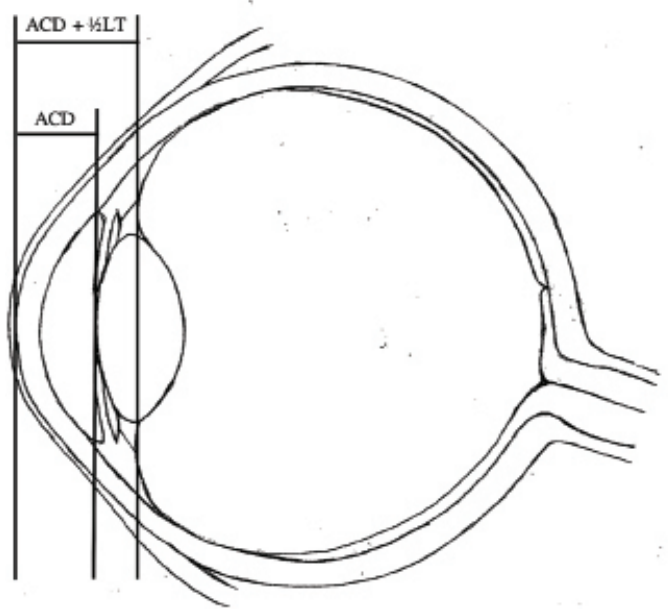

Fig. 1. Proposed ELP prediction model. The mid-sagittal plane of the natural lens is estimated to be positioned at half the value of its thickness from its anterior surface. This is hypothesised to align with the principal plane of the non-angulated biconvex $\mathrm{OOL}$ within the capsular bag. 
Improving the prediction of effective lens position for IOL power calculations

Table 1. Post-operative IOL ELP prediction parameters

\begin{tabular}{|l|l|l|}
\hline Current & $A C D$ & $\begin{array}{l}\text { The distance from the anterior surface of the cornea to } \\
\text { the anterior surface of the IOL }\end{array}$ \\
\hline Proposed & $A C D+1 / 2 \mathrm{LT}$ & $\begin{array}{l}\text { The distance from the anterior surface of the cornea to the } \\
\text { mid-sagittal plane positioned at half the thickness of the } \\
\text { IOL }\end{array}$ \\
\hline
\end{tabular}

\section{Materials and methods}

This study has received approval from the Northern Sydney Local Health District Human Research Ethics Committee and is in accordance with the National Health and Medical Research Council's National Statement on Ethical Conduct in Human Research 2007 and the CPMP/ICH Note for Guidance on Good Clinical Practice. This is a single-centre project, which has been assessed as low/negligible risk.

\section{Sample and data collection}

This retrospective cohort study utilised pre- and post-operative data obtained from 162 consecutive cases of phacoemulsification and implantation of posterior-chamber IOL (Alcon SN60WF). Ocular biometric measurements for each case were obtained pre-operatively ( 1 week before surgery) and post-operatively (2 months after surgery) using the IOLMaster 700 with SWEPT source biometry (Carl Zeiss Meditec, Jena, Germany).

\section{Inclusion and exclusion criteria}

Cases of phacoemulsification with posterior-chamber IOL implantation performed by Dr. Keith Ong, from the period of 1 August 2017 until 28 February 2018, were included. Patients who had undergone previous eye surgery, previous trauma to the eye or incomplete pre- and post-operative data were excluded from this study. The Alcon SN60WF implant, a biconvex IOL with planar (non-angulated) haptics, was used for all cases.

\section{Statistical methods}

Predictions made using ACD were compared to those made using the proposed parameter, $A C D+1 / 2 L T$, for each case (Table 1). Residual analyses were performed for both prediction parameters. Average model ELP prediction error was compared through root-mean-square-error (RMSE) and coefficient of determination $\left(R^{2}\right)$ statistics.

The ELP prediction error $\left(\mathrm{PE}_{\mathrm{ELP}}\right)$ was determined by calculating the difference between the predicted ELP and the measured ELP post-operation for the two parameters:

$$
P E_{E L P}=A C D_{\text {pre-op }}-A C D_{\text {post-op }}
$$




$$
P E_{E L P}=A C D+1 / 2 L T_{\text {pre-op }}-A C D+1 / 2 L T_{\text {post-op }}
$$

To permit comparison of data obtained from eyes of different ALs, PE $E_{E L P}$ was compared as a percentage of the measured ELP to account for proportional bias:

$$
\begin{aligned}
\text { Percentage } P E_{E L P} & =\frac{P E_{A C D}}{A C D_{\text {post-op }}} \\
\text { Percentage } P E_{E L P} & =\frac{P E_{A C D+1 / 2 T}}{A C D+1 / 2 L T_{\text {post-op }}}
\end{aligned}
$$

Paired two-tailed $t$-tests were performed $(a=0.05)$ for mean $\mathrm{PE}_{\mathrm{ELP}}$ and mean percentage $\mathrm{PE}_{\mathrm{ELP}}$. One-tailed one-sample $t$-tests were performed $(a=0.05)$ to determine directionality of $\mathrm{PE}_{\mathrm{ELP}}$ for predictions derived from each parameter.

Data collected were entered into Microsoft Excel 2010 (Microsoft Corporation, Redmond, WA, USA). All analyses were performed with aid of software, GraphPad Prism 7 for Windows (GraphPad Software, La Jolla, CA, USA, www.graphpad.com). Prior to applying parametric tests, D'Agostino-Pearson normality tests ${ }^{10}$ were applied to all data sets.

\section{Results}

\section{Study sample}

A total of 162 consecutive cases (from 100 patients), undergoing phacoemulsification and implantation of posterior-chamber IOL (AlconSN60WF) during the 6-month study period, were analysed. Of these, 38 cases belonged to patients who undertook surgery on one eye and 124 cases to patients on both. There were 73 eyes of male patients (45\%). Mean patient age was $67.8 \pm 5.28$ years (range, 46 to 83 years) (Table 2). The mean AL was $24.68 \pm 1.89 \mathrm{~mm}$ (range, 21.72 to $32.99 \mathrm{~mm}$ ).

Table 2. Patient characteristics

\begin{tabular}{|l|l|}
\hline Variable & \\
\hline No. of cases (patients) & $162(100)$ \\
\hline One eye & $38(38)$ \\
\hline Both eyes & $124(62)$ \\
\hline Male & $73(45)$ \\
\hline Female & $89(55)$ \\
\hline Age (years) & \\
\hline Mean \pm SD & $67.8 \pm 5.28$ \\
\hline Range & $46-83$ \\
\hline
\end{tabular}


Improving the prediction of effective lens position for IOL power calculations

Table 3. Characteristics of study sample

\begin{tabular}{|l|l|l|l|l|}
\hline \multirow{2}{*}{ Variable } & \multicolumn{2}{l|}{ Pre-operation (1 week) } & \multicolumn{2}{l|}{ Post-operation (2 months) } \\
\cline { 2 - 5 } & Mean \pm SD $(\mathbf{m m})$ & SEM & Mean \pm SD $(\mathbf{m m})$ & SEM \\
\hline ACD & $3.02 \pm 0.33$ & 0.02 & $4.59 \pm 0.29$ & 0.02 \\
\hline LT & $4.69 \pm 0.36$ & 0.03 & $0.61 \pm 0.10$ & 0.01 \\
\hline AL & $24.68 \pm 1.89$ & 0.15 & & \\
\hline
\end{tabular}

Ocular biometric measurements were made using IOLMaster 700 (Zeiss, Australia), pre-operatively ( 1 week) and post-operatively ( 2 months) for all eyes analysed ( $n=162$ ).

SEM, standard error of mean.

A summary of the characteristics for eyes analysed are included in Table 3 . Normality tests supported that sampled data, ELP prediction errors and pairs were sampled from a population where differences are consistent with a normal distribution $(\mathrm{p}<0.05)$.

\section{Residual error analysis}

The RMSE performance and $R^{2}$ statistic for ACD and ACD + 1/2LT predictions are presented in Figure 2. Performance metrics indicate that the proposed prediction parameter achieves a reduced average model prediction error $(\mathrm{RMSE}=0.50$, $\left.R^{2}=0.70\right)$, as compared to ACD (RMSE $=1.57 \mathrm{~mm}, R^{2}=0.67$ ) (Fig. 2).

\section{Analysis of variance and hypothesis testing}

The mean $\mathrm{PE}_{\mathrm{ELP}}$ for predictions were $-1.57 \pm 0.20 \mathrm{~mm}$ for those derived from $\mathrm{ACD}+$ $1 / 2 \mathrm{LT}$ and $0.48 \pm 0.16 \mathrm{~mm}$ for those derived from ACD (Fig. 3a and Table 4). The difference between means of $2.04 \pm 0.18 \mathrm{~mm}$ between two prediction groups was statistically significant $(95 \% \mathrm{Cl}=2.02-2.07 \mathrm{~mm} ; t=142.3, \mathrm{df}=161 ; \mathrm{p}<0.0001$; Table 5).

Comparing $\mathrm{PE}_{\mathrm{ELP}}$ as a percentage of the measured ELP to account proportional bias due to different $A L$ of eyes, predictions made based on ACD alone demonstrated a mean $\mathrm{PE}_{\mathrm{ELP}}$ of $-34.22 \% \pm 4.78 \%$, while predictions made using the proposed algorithm yielded a $\mathrm{PE}_{\mathrm{ELP}}$ of $9.88 \% \pm 3.50 \%$ (Fig. $3 \mathrm{~b}$ and Table 4). Applying paired two-tailed $t$-test analysis, a mean of differences of $44.10 \% \pm$ $5.22 \%$ was found to be statistically significant $(95 \% \mathrm{Cl}=43.30 \%-44.91 \% ; t=107.6$, $\mathrm{df}=161 ; \mathrm{p}<0.0001)($ Table 5$)$.

\section{Direction of error}

One-tailed one-sample $t$-test analyses showed that predictions made using ACD uniformly underestimated outcomes $(95 \% \mathrm{Cl}=-1.60$ to $-1.53 \mathrm{~mm} ; t=-99.27$, $\mathrm{df}=161 ; \mathrm{p}<0.0001)$, whereas predictions made using ACD $+1 / 2 \mathrm{LT}$ uniformly overestimated $\operatorname{ELP}(95 \% \mathrm{Cl}=0.45-0.50 \mathrm{~mm} ; t=39.04, \mathrm{df}=161 ; \mathrm{p}<0.0001)$ (Fig. 3). Both ELP prediction parameters demonstrated directionality in respective degrees of $P E_{E L P}$. 


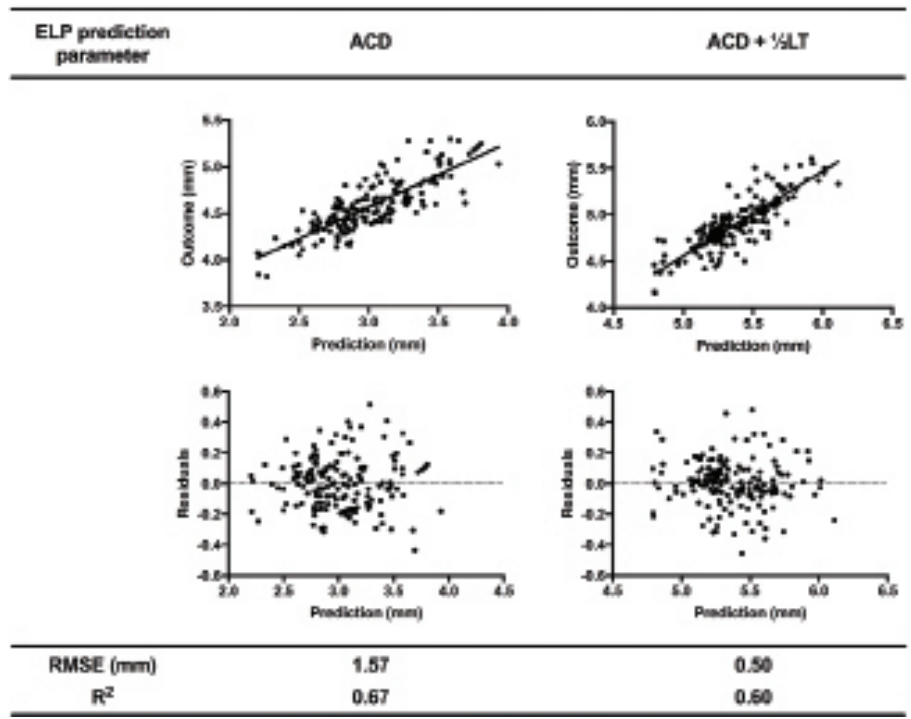

Fig. 2. Predicted vs. observed ELP ( $\mathrm{mm}$ ) (top) and graphs of residuals (bottom). RMSE and R2 were used to evaluate average ELP prediction error.

\section{Discussion}

The results of the present study demonstrate that improved accuracy for ELP predictions may be achieved using proposed parameter, $A C D+1 / 2 L T$, as compared to use of the ACD measurement alone. This thereby supports the hypothesis that accounting for half the value of LT improves estimations of the post-operative position of the IOL principal plane. Overall, a significant improvement in average error was achieved with the proposed prediction parameter (RMSE $=0.5 \mathrm{~mm}$ ),
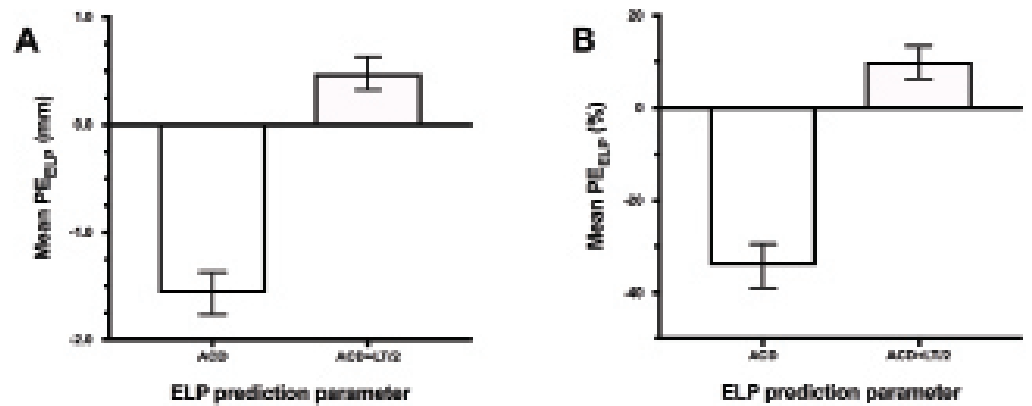

Fig. 3. Mean error of ELP predictions ( $n=162$ ). (a) The mean difference between the predicted ELP and the measured outcome. (b) The mean prediction error is shown as a percentage of the measured ELP post-operation to permit comparison between eyes of different ALs. Error bars represent standard deviation. 
Improving the prediction of effective lens position for IOL power calculations

Table 4. Comparison of post-operative ELP predictability for ACD alone and proposed parameter $(n=162)$

\begin{tabular}{|l|l|l|}
\hline ELP prediction parameter & ACD & ACD $+1 / 2 \mathrm{LT}$ \\
\hline Mean $\mathrm{PE}_{\mathrm{ELP}} \pm \mathrm{SD}(\mathrm{mm})$ & $-1.56 \pm 0.20$ & $0.48 \pm 0.16$ \\
\hline $\mathrm{SEM}$ & 0.02 & 0.01 \\
\hline Mean $\mathrm{PE}_{\mathrm{ELP}} \pm \mathrm{SD}(\%)$ & $-34.22 \pm 4.78$ & $9.88 \pm 3.50$ \\
\hline $\mathrm{SEM}$ & 0.38 & 0.27 \\
\hline
\end{tabular}

p-Value $<0.05$ was statistically significant.

compared to ACD (RMSE $=1.57 \mathrm{~mm}$ ). Accounting for half the value of $\mathrm{LT}$ in addition to $A C D$ resulted in a significant reduction in mean $P E_{E L P}$ compared to using ACD alone, from $-1.57 \pm 0.20 \mathrm{~mm}$ to $0.48 \pm 0.16 \mathrm{~mm}$ (Fig. 2 and Table 5).

Currently, IOL power calculations are adjusted to minimise systematic error by the use of fudge factors and IOL constant optimisation processes ${ }^{11}$; however, these measures only improve prediction accuracy on average, by minimising error for eyes within a target range, but does not cater for the non-average eye. For this reason, $\mathrm{IOL}$ constants are known to vary with $\mathrm{AL}$, and there is at present no single formula that can optimally predict IOL power for all eyes. ${ }^{12,13}$ The success in reducing error by such means also depend in practice, on the baseline used for optimisation that varies with the patient cohort and approach; Haigis, for example, recommends the use of data from at least 50 eyes for optimisation. ${ }^{14}$ In contrast, the proposed parameter should facilitate individualisation of ELP predictions by accounting for variations in LT. Measurements of ACD to the anterior surface of the lens are affected by LT, which the proposed parameter aims to overcome. By determining the position of the mid-sagittal plane of a biconvex lens, the effect of variations in thickness of phakic lenses on ELP predictions, are minimised.

While our results demonstrate a significant reduction in error for the proposed

Table 5. Paired two-tailed t-test showing significance of differences between the means of PE resulting from the current and proposed ELP prediction parameters $(n=162)$

\begin{tabular}{|l|l|l|l|l|l|}
\hline & $\begin{array}{l}\text { Mean of } \\
\text { differences } \\
(\mathbf{m m})\end{array}$ & $\begin{array}{l}\text { SEM of } \\
\text { differences }\end{array}$ & p-Value & \multicolumn{2}{|l|}{ 95\% Confidence interval } \\
\cline { 4 - 6 } & $2.04 \pm 0.18$ & 0.01 & $<0.0001$ & 2.02 & 2.07 \\
\hline $\begin{array}{l}\text { Mean PE } \\
\text { SD (mm) }\end{array}$ & & & $<0$ Ler limit & Upper limit \\
\hline $\begin{array}{l}\text { Mean PE } \\
\text { SD (\%) }\end{array}$ & $44.10 \pm 5.22$ & 0.41 & $<0.0001$ & 43.30 & 44.91 \\
\hline
\end{tabular}

p-Value $<0.05$ was considered statistically significant. 
parameter, they have not been eliminated. We acknowledge that while our model assumes that the IOL is perfectly biconvex, the human lens is not; the anterior surface tends to be more planar than the posterior. ${ }^{15}$ With further analysis, however, our results demonstrate that $P E_{E L P}$ observed with the use of $A C D+1 / 2 L T$ occurs in a single direction, as do predictions made using ACD. This residual error can therefore be systematically accounted for using current approaches; correction factors and optimisation of IOL constants used in current IOL power calculation formulae may still be applied.

Above all, our results also show a reduction in the spread of $\mathrm{PE}_{\mathrm{ELP}}$, indicating that ELP predictions were made with greater precision using the proposed parameter ( $3.50 \% \mathrm{SD})$, compared to using the ACD measurement alone $(4.78 \%$ $\mathrm{SD})$. This finding may hold greater implications than the reduction of error previously discussed, as while constant optimisation processes and fudge factors may correct for average error, the reliability of predictions (the dispersion of error around the mean) are unchanged by such measures. ${ }^{16}$

Overall, this preliminary study has demonstrated the potential for prediction of ELP based on the position of the mid-sagittal plane of the natural lens in the phakic eye to reduce inaccuracy and imprecision, compared to ACD alone. Despite statistically promising results, the potential and scope for clinical application of the proposed ELP prediction parameter will require further study. In this regard, the addition of half the value of LT represents a simple modification to current ELP predictions based on the ACD measurement, which may easily be applied to existing IOL power calculation formulae and constant optimisation processes. Additionally, IOL formulae using ACD, such as the Haigis formula, cannot be used in the pseudophakic eye when IOL exchange is required, while our proposed parameter would allow usage of such formulae. Finally, while the scope of the present study extends only to predicting the ELP of planar haptic IOL, applying the proposed model to angled haptic IOLs may be possible with a correction factor. It may thus be of interest for future studies to evaluate refractive outcomes when applying the proposed parameter, as well as its performance when applied to pseudophakic eyes and angulated haptics.

\section{Conclusion}

Our small study demonstrated that the IOL ELP can more accurately and reliably be determined using ACD $+1 / 2 \mathrm{LT}$, as compared to $A C D$ alone. The incorporation of the proposed parameter into current IOL power calculation formulae may therefore improve the predictability of post-operative refraction and thereby minimise refractive surprise in cataract surgery. In light of our present findings, we hope to explore the clinical applications of the proposed ELP prediction parameter in future studies. 


\section{References}

1. Norrby SJ. Sources of error in intraocular lens power calculation. Cataract Refract Surg. 2008;34(3):368-376.

2. Muthappan V, Paskowitz D, Kazimierczak A, Jun AS, Ladas J, Kuo IC. Measurement and use of postoperative anterior chamber depth of fellow eye in refractive outcomes. J Cataract Refract Surg. 2015;41(4):778-784. doi:10.1016/j.jcrs.2014.08.034.

3. Rosen AM, Denham DB, Fernandez V, et al. In vitro dimensions and curvatures of human lenses. Vision Research. 2006;46(6-7):1002-1009.

4. Jones $C E$, Atchison DA, Pope JM. Changes in lens dimensions and refractive index with age and accommodation. Optom Vis Sci. 2007;84(10):990-995.

5. Richdale K, Bullimore MA, Zadnik K. Lens thickness with age and accommodation by optical coherence tomography. Ophthalmic Physiol Opt. 2008;28(5):441-447.

6. Sheard R. Optimising biometry for best outcomes in cataract surgery. Eye. 2014;28(2):118-125.

7. Ong K, Feng L. Prevalence of variation in predicted refraction between different intraocular lens formulae. Asian J Opthalmol. 2018;16:60-61.

8. Abulafia A, Barrett GD, Rotenberg M, et al. Intraocular lens power calculation for eyes with an axial length greater than $26.0 \mathrm{~mm}$ : comparison of formulas and methods. J Cataract Refract Surg. 2015;41(3):548-556. doi:10.1016/j.jcrs.2014.06.033.

9. Wong RS, Ong K. Influence of anterior chamber depth on postoperative refractive outcome in Chinese eyes. Asian J Opthalmol. 2018;16:79-85.

10. D'Agostino RB. Tests for normal distribution. In: D'Agostino RB, Stepenes MA, eds. GoodnessOf-Fit Techniques. Boca Raton, FL: CRC Press.

11. Aristodemou P, Knox Cartwright NE, Sparrow JM, Johnston RL. Intraocular lens formula constant optimization and partial coherence interferometry biometry: refractive outcomes in 8108 eyes after cataract surgery. J Cataract Refract Surg. 2011;37(1):50-62.

12. Sanders DR, Retzlaff J, Kraff MC. Comparison of the SRK II formula and other second generation formulas. J Cataract Refract Surg. 1988;14(2):136-141.

13. Sahin A, Hamrah P. Clinically relevant biometry. Curr Opin Ophthalmol. 2012;23(1):47-53.

14. Haigis W. User group for laser interference biometry. http://www.augenklinik.uni-wuerzburg.de/ ulib/czm/dload.htm. Accessed 9 September 2013.

15. Dubbelman M, Van der Heijde GL. The shape of the ageing human lens: curvature, equivalent refractive index and the lens paradox. Vision Research. 2001;41(14):1867-1877.

16. Olsen T. Improved accuracy of intraocular lens power calculation with the Zeiss IOLMaster. Acta Ophthalmologica Scandinavica. 2007;85(1):84-87. 